\title{
Prospects for immunotherapy of acute myeloid leukemia using $\gamma \delta$ T cells
}

\author{
"Perhaps the time is now right to evaluate $\gamma \delta$ T-cell \\ immunotherapy in patients with acute myeloid leukemia, \\ particularly in that large majority of patients who are unsuitable \\ for allogeneic hematopoietic stem cell transplantation."
}

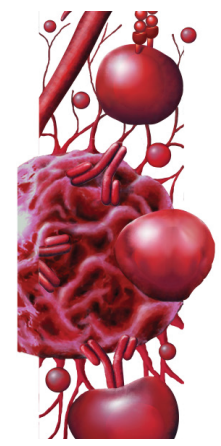

First draft submitted: 5 December 2016; Accepted for publication: 15 December 2016; Published online: 27 January 2017

Keywords: $\gamma \delta$ T cell $\bullet$ acute myeloid leukemia • adoptive cell therapy

The majority of $\mathrm{T}$ lymphocytes express an $\alpha \beta$ T-cell receptor (TCR) that detects processed peptide antigen, presented in the context of MHC antigens. However, a significant minority population (generally $<10 \%$ ) express an alternative TCR, comprising $\gamma$ and $\delta$ subunits. The distinctive nature of these cells has been appreciated since their discovery and initial characterization, in the early to mid-1980s. Activation of $\gamma \delta \mathrm{T}$ cells is commonly triggered by engagement of diverse molecular markers of cellular stress or transformation. Consistent with this, the interrelationship between these elusive $T$ cells and cancer has preoccupied translational investigators for several years. Early studies demonstrated that $\gamma \delta$ T cells undergo oligoclonal expansion in patients with malignant disease, can infiltrate neoplastic lesions and provide immune surveillance against tumor formation in mice [1]. Remarkably, the presence of intratumoral $\gamma \delta \mathrm{T}$ cells has recently been ranked as the single most prognostically favorable immune-cell infiltrate identified across a panel of 39 tumor types [2]. Furthermore, the MHC unrestricted nature of antigen recognition by these cells means that they do not cause graft versus host disease, theoretically facilitating their safe 'off-the-shelf' or allogeneic use. Balancing this however, $\gamma \delta$ T cells sometimes act as willing conspirators in the malignant process - an event that may be accompanied by IL-17 release and/or deployment of one or more immunosuppressive functions, such as galectin-1 release $[3,4]$. These considerations highlight the challenge imposed to effectively harness these cells for immunotherapeutic purposes.

$\gamma \delta \mathrm{T}$ cells may be viewed as the ultimate multi-taskers or 'jack of all trades' of the immune system. They straddle innate and adaptive immunity, and display features that are reminiscent of professional antigen-presenting cells, natural killer (NK) cells, conventional cytotoxic $\alpha \beta \mathrm{T}$ cells and/or suppressive regulatory $\mathrm{T}$ cells. Once activated, $\gamma \delta$ T cells deploy several effector mechanisms to destroy target cells, including the release of perforin and granzymes, inflammatory cytokines (e.g., IFN- $\gamma$ and TNF- $\alpha$ ), TRAIL and Fas ligand. These cells also provide an integrated and anatomically compartmentalized surveillance system that operates within the bloodstream and the tissues. In man, these two roles are broadly served by subsets that respectively express a $\gamma \delta$ TCR containing a $V \delta 2$ or a non-V $\delta 2$ subunit (most commonly either V $\delta 1, V \delta 3$ or V $\delta 5$ ). However, these distinctions do not appear to be absolute. For example, although $\mathrm{V} \delta 1^{+} \mathrm{T}$ cells are generally the dominant $\gamma \delta$ tumor-infiltrating population in melanomas, it is the proportion of infiltrating $\mathrm{V} \delta 2^{+}$(but not $\mathrm{V} \delta 1^{+}$) $\mathrm{T}$ cells that correlates with earlier disease stage and reduced risk of metastatic spread [5]. Conversely, enhanced reconstitution of V 81 enriched $\gamma \delta$ T cells following allogeneic hematopoietic stem cell transplantation (aHSCT)
Leena Halim', Ana

Catarina Parente-Pereira ${ }^{1}$ \& John Maher ${ }^{*}, 1,2,3$

'CAR Mechanics Group, Division of Cancer Studies, King's College London, Guy's Hospital Campus, Great Maze Pond, London SE1 9RT, UK ${ }^{2}$ Department of Clinical Immunology \& Allergy, King's College Hospital NHS Foundation Trust, London, UK ${ }^{3}$ Department of Immunology, Eastbourne Hospital, East Sussex, UK *Author for correspondence: Tel.: +442071881468 Fax: +442071880919 john.maher@kcl.ac.uk 
is associated with improved survival in patients with acute myeloid leukemia (AML) [6]. Underscoring this complexity, it has recently been proposed that diverse butyrophilin-like molecules regulate the maturation and expansion of locally operative $\gamma \delta$ T-cell subtypes in distinct epithelial tissues [7]. For these reasons, both $\mathrm{V} \delta 2^{+}$and non-V $\delta 2^{+}$subsets warrant consideration as therapeutic effectors against malignant disease. The focus of this editorial is to assess prospects for $\gamma \delta$ T-cell immunotherapy of AML - a disorder that continues to increase worldwide and which accounted for approximately 265,461 deaths in 2012 [8].

In the majority of AML patients, disease remission can be achieved using combination chemotherapy. Curative consolidation is then achieved most effectively using a cellular immunotherapy, namely aHSCT. Unfortunately, this option is unavailable to most patients, either due to lack of a suitable donor or comorbidity and risk of transplantation-related mortality. It is logical therefore that investigators have sought to develop alternative cellular immunotherapies that combine this level of therapeutic potency with improved safety and universality of application. Against this background, it is opportune to ask how well $\gamma \delta$ T cells might measure up to this task?

The best studied $\gamma \delta$ subset is that which is most accessible, namely the V $\gamma 9 \mathrm{~V} \delta 2$ (also termed V $\gamma 2 \mathrm{~V} \delta 2$ ) $\mathrm{T}$ cells that predominate in the bloodstream. These cells recognize phosphoantigen $(\mathrm{PAg})$ intermediates of the mevalonate pathway, notably isopentenyl pyrophosphate. Given that aberrant mevalonate metabolism contributes directly to transformation [9], it is not surprising that increased flux through this pathway leading to enhanced PAg production is commonly found in AML blasts [10]. Accumulation of PAg is further potentiated by aminobisphosphonate agents, such as zoledronic acid, accounting for its propensity to cause transient expansion of these cells and febrile reactions/ flu-like symptoms in many patients [11]. Recognition of PAg is dependent upon butyrophilin-3A1, although additional intermediates appear to be required to fully reconstruct this antigen-presenting machinery.

The ability of $\mathrm{V} \gamma 9 \mathrm{~V} \delta 2 \mathrm{~T}$ cells to detect leukemic and other abnormal cell types is strengthened by their expression of a series of complementary innate receptors, many of which are shared with NK cells. Preeminent among these is NKG2D, a C-type lectin that binds the stress ligands, MICA/B and ULBP family members. V $\gamma 9 \mathrm{~V} \delta 2 \mathrm{~T}$ cells also express DNAM-1, which interacts with nectin-2 (CD112) and polio virus receptor (nectin-like 5; CD155).

Evidence that $\mathrm{V} \gamma 9 \mathrm{~V} \delta 2 \mathrm{~T}$ cells exert meaningful control over AML is supported by a number of clinical observations. The combination of zoledronic acid and low dose IL-2 was administered to patients with a range of refractory malignancies, with the goal of activating the endogenous $\mathrm{V} \gamma 9 \mathrm{~V} \delta 2 \mathrm{~T}$-cell population. Notably, the only individuals that benefited from this intervention had refractory/relapsed AML (two partial responses and two stable disease in seven evaluable patients) [12]. Small scale studies in which haploidentical $\gamma \delta \mathrm{T}$ cells and zoledronic acid have been infused in patients with AML and other hematologic malignancies have also resulted in disease remission [13].

Preclinical evidence also provides support for this contention. Leukemic blasts express ligands for NKG2D and DNAM-1 and can be killed in vitro by $\mathrm{V} \gamma 9 \mathrm{~V} \delta 2 \mathrm{~T}$ cells [14,15]. Furthermore, $\mathrm{V} \gamma 9 \mathrm{~V} \delta 2 \mathrm{~T}$ cells traffic to bone marrow and have been shown to retard progression of an aggressive AML xenograft [14]. Blocking studies using antibodies and mevalonate pathway inhibitors indicate that TCR and DNAM-1 engagement are the most important interactions in triggering the killing of AML cells [14,15]. By contrast, NKG2D ligands, such as MICA/B and ULBP family members are generally expressed at lower levels on leukemic blasts. Nonetheless, ULBP-1 expression predicts sensitivity of AML cells to killing by allogeneic V $\gamma 9 \mathrm{~V} 82 \mathrm{~T}$ cells, whereas nectin-2 expression correlated negatively in this regard [16].

Non-V $\delta 2 \gamma \delta \mathrm{T}$ cells are primarily resident within epithelial tissues where they protect against infection (including viral reactivation) and transformation [4]. Many are responsive to stress ligands, cytomegalovirus (CMV) and other viruses, while some cross-react with poorly characterized tumor-associated molecules that include endothelial protein $C$ receptor. Other non-V $\delta 2$ cells engage glycolipid antigens that are presented on tumor and other cell types by CD1 family members, notably CD1c and CD1d [5]. Adding to complexity, hybrid receptors have also been described in which V $\delta 1$ segments are fused with segments from the TCR $\alpha$ chain family.

CMV-responsive V $\delta 1$ cells are commonly increased in patients following aHSCT, owing to CMV reactivation. Intriguingly, this is associated with reduced relapse of disease, suggesting that these cells also exert intrinsic antileukemic activity [15]. In keeping with this, V $\delta 1 \gamma \delta$ Tcells also demonstrate in vitro cytotoxic activity against AML cells and this is further enhanced following CMV reactivation [15,17]. While molecular interactions remain to be fully characterized, suspicion falls on the TCR itself in addition to the broad array of innate receptors expressed by these cells, including NKG2D, DNAM-1 and NK receptors that include NKp30, NKp44 and NKp46 [18].

Pathways forward can be envisioned for the clini-

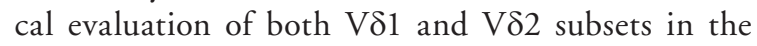


experimental immunotherapy of patients with AML. Vy9V82 $\mathrm{T}$ cells are traditionally considered to be easier to expand in vitro, although methods have recently been described to allow efficient, convenient and clinically compatible expansion of $\mathrm{V} \delta 1^{+} \mathrm{T}$ cells from peripheral blood [19]. Furthermore, V $\delta 1$ cells are reported to be more resistant to activation-induced cell death than their V $\delta 2$ counterparts [20]. Balancing this however, $\mathrm{V} \gamma 9 \mathrm{~V} \delta 2 \mathrm{~T}$ cells have a natural propensity to traffic to sites of disease in AML. Furthermore, immunotherapy using these cells can be potentiated by aminobisphosphonates, such as zoledronic acid. These agents sensitize target cells to attack by $\mathrm{V} \gamma 9 \mathrm{~V} \delta 2 \mathrm{~T}$ cells and are naturally tropic for bone, creating a drug reservoir close to the site of disease origin. Further enhancement of aminobisphosphonate delivery to sites of malignant disease can be achieved using liposomeencapsulated formulations, which exploit the abnormally leaky neovasculature that is found in many solid tumors and hematological malignancies [21]. Providing additional therapeutic options, $\alpha \beta$ T cells can be genetically engineered to express $\gamma \delta$ TCRs of either the $V \delta 1$ or $V \delta 2$ subtype, thereby conferring their antileukemic specificity upon these cells [17]. To date, most clinical trials of $\gamma \delta$ T-cell immunotherapy have focused on solid tumors, where efficacy has been limited. This contrasts with their better-studied first

\section{References}

1 Girardi M, Oppenheim DE, Steele CR et al. Regulation of cutaneous malignancy by $\gamma \delta$ T cells. Science 294(5542), 605-609 (2001).

2 Gentles AJ, Newman AM, Liu CL et al. The prognostic landscape of genes and infiltrating immune cells across human cancers. Nat. Med. 21(8), 938-945 (2015).

3 Daley D, Zambirinis CP, Seifert L et al. $\gamma \delta \mathrm{T}$ cells support pancreatic oncogenesis by restraining alphabeta $\mathrm{T}$ cell activation. Cell 166(6), 1485-1499 e1415 (2016).

4 Rutkowski MR, Stephen TL, Svoronos N et al. Microbially driven TLR5-dependent signaling governs distal malignant progression through tumor-promoting inflammation. Cancer Cell 27(1), 27-40 (2015).

5 Toia F, Buccheri S, Anfosso A et al. Skewed differentiation of circulating V $\gamma 9 \mathrm{~V} \delta 2 \mathrm{~T}$ lymphocytes in melanoma and impact on clinical outcome. PLoS ONE 11(2), e0149570 (2016).

6 Godder KT, Henslee-Downey PJ, Mehta J et al. Long term disease-free survival in acute leukemia patients recovering with increased $\gamma \delta$ T cells after partially mismatched related donor bone marrow transplantation. Bone Marrow Transplant. 39(12), 751-757 (2007).

7 Di Marco Barros R, Roberts NA, Dart RJ et al. Epithelia use butyrophilin-like molecules to shape organ-specific $\gamma \delta$ T cell compartments. Cell 167(1), 203-218 e217 (2016). cousins, NK cells, which have been extensively studied for their antileukemic activity. Perhaps the time is now right to evaluate $\gamma \delta \mathrm{T}$-cell immunotherapy in patients with AML, particularly in that large majority of patients who are unsuitable for aHSCT.

\section{Financial \& competing interests disclosure}

Research on $\gamma \delta$ T-cell immunotherapy in the authors' laboratory is supported by the Medical Research Council, Cancer Research UK, Bayer HealthCare, Bloodwise, the King's Health Partners Research and Development Fund, British Council Newton Fund Institutional Links Award, the Experimental Cancer Medicine Centre at King's College London and by the National Institute for Health Research (NIHR) Biomedical Research Centre based at Guy's and St Thomas' NHS Foundation Trust and King's College London. The views expressed are those of the authors and not necessarily those of the NHS, the NIHR or the Department of Health. J Maher is chief scientific officer of Leucid Bio, a spinout company focused on CAR T-cell and $\mathrm{V} \gamma 9 \mathrm{~V} \delta 2 \mathrm{~T}$-cell immunotherapies for malignant disease. The authors have no other relevant affiliations or financial involvement with any organization or entity with a financial interest in or financial conflict with the subject matter or materials discussed in the manuscript apart from those disclosed.

No writing assistance was utilized in the production of this manuscript.

8 Union for International Cancer Control. www.who.int/ selection_medicines/committees/expert/20/applications/ AML_APL.pdf

9 Clendening JW, Pandyra A, Boutros PC et al. Dysregulation of the mevalonate pathway promotes transformation. Proc. Natl Acad. Sci. USA 107(34), 15051-15056 (2010).

10 Pandyra A, Mullen PJ, Kalkat M et al. Immediate utility of two approved agents to target both the metabolic mevalonate pathway and its restorative feedback loop. Cancer Res. 74(17), 4772-4782 (2014).

11 Kunzmann V, Bauer E, Wilhelm M. $\gamma / \delta$ T-cell stimulation by pamidronate. N. Engl. J. Med. 340(9), 737-738 (1999).

12 Kunzmann V, Smetak M, Kimmel B et al. Tumor-promoting versus tumor-antagonizing roles of $\gamma \delta \mathrm{T}$ cells in cancer immunotherapy: results from a prospective Phase I/II trial. $J$. Immunother. 35(2), 205-213 (2012).

13 Wilhelm M, Smetak M, Schaefer-Eckart K et al. Successful adoptive transfer and in vivo expansion of haploidentical $\gamma \delta \mathrm{T}$ cells. J. Transl. Med. 12, 45 (2014).

14 Gertner-Dardenne J, Castellano R, Mamessier E et al. Human $\mathrm{V} \gamma 9 \mathrm{~V} \delta 2 \mathrm{~T}$ cells specifically recognize and kill acute myeloid leukemic blasts. J. Immunol. 188(9), 4701-4708 (2012).

15 Airoldi I, Bertaina A, Prigione I et al. $\gamma \delta$ T-cell reconstitution after HLA-haploidentical hematopoietic transplantation depleted of TCR-alphabeta+/CD19+ lymphocytes. Blood 125(15), 2349-2358 (2015). 
16 Gundermann S, Klinker E, Kimmel B et al. A comprehensive analysis of primary acute myeloid leukemia identifies biomarkers predicting susceptibility to human allogeneic Vy9V82 T cells. J. Immunother. 37(6), 321-330 (2014).

17 Scheper W, van Dorp S, Kersting $S$ et al. $\gamma \delta \mathrm{T}$ cells elicited by CMV reactivation after allo-SCT cross-recognize CMV and leukemia. Leukemia 27(6), 1328-1338 (2013).

18 Correia DV, Fogli M, Hudspeth K, Da Silva MG, Mavilio D, Silva-Santos B. Differentiation of human peripheral blood V $\delta 1+T$ cells expressing the natural cytotoxicity receptor NKp30 for recognition of lymphoid leukemia cells. Blood 118(4), 992-1001 (2011).
19 Almeida AR, Correia DV, Fernandes-Platzgummer A et al. Delta one $T$ cells for immunotherapy of chronic lymphocytic leukemia: clinical-grade expansion/differentiation and preclinical proof of concept. Clin. Cancer Res. 22(23), 5795-5804 (2016).

20 Siegers GM, Lamb LS Jr. Cytotoxic and regulatory properties of circulating $\mathrm{V} \delta 1+\gamma \delta \mathrm{T}$ cells: a new player on the cell therapy field? Mol. Ther. 22(8), 1416-1422 (2014).

21 Parente-Pereira AC, Shmeeda H, Whilding LM et al. Adoptive immunotherapy of epithelial ovarian cancer with Vy9V82 $\mathrm{T}$ cells, potentiated by liposomal alendronic acid. $J$. Immunol. 193(11), 5557-5566 (2014). 